\title{
Inference for a Class of Transformed Hazards Models
}

\author{
DONGLIN ZENG, GUOSHENG YIN and JOSEPH G. IBRAHIM
}

We consider a new class of transformed hazard rate models. This class contains both the multiplicative hazards model and the additive hazards model as special cases. The sieve maximum likelihood estimators are derived for the model parameters and the estimators for the regression coefficients are shown to be consistent and asymptotically normal with variance achieving the semiparametric efficiency bound. Simulation studies are conducted to examine the small-sample properties of the proposed estimates and a real dataset is used to illustrate our approach.

KEY WORDS: Box-Cox transformation; Sieve estimation; Transformed hazard rate; Wavelet approximation

Donglin Zeng is Assistant Professor and Joseph G. Ibrahim is Professor, Department of Biostatistics, CB\# 7420, University of North Carolina, Chapel Hill, NC 27599-7420. Guosheng Yin is Assistant Professor, Department of Biostatistics and Applied Mathematics, M. D. Anderson Cancer Center, The University of Texas, Houston, TX 77030-4009. We are grateful to the two referees for their critical and helpful comments. 


\section{Introduction}

In survival analysis, the Cox (Cox, 1972) multiplicative hazards model has been extensively used. In this model, the hazard rate function of the survival time given an external (possibly time-dependent) covariate vector $\mathbf{Z}(t)$ is assumed to be

$$
\lambda(t \mid \mathbf{Z}(t))=\lambda(t) \exp \left\{\boldsymbol{\beta}^{T} \mathbf{Z}(t)\right\}
$$

where $\lambda(t)$ is an unknown and unspecified baseline hazard function and $\boldsymbol{\beta}$ is the regression coefficient for $\mathbf{Z}(t)$. An efficient estimate for $\boldsymbol{\beta}$ can be obtained by maximizing a partial likelihood function (Cox, 1975; Andersen and Gill, 1982). Since the proportionality in the multiplicative hazards model does not hold in many applications, one alternative form to model the hazard rate function is to assume that the hazard risks are additive across covariates; i.e.,

$$
\lambda(t \mid \mathbf{Z}(t))=\mu(t)+\boldsymbol{\beta}^{T} \mathbf{Z}(t)
$$

where $\mu(t)$ is an unknown baseline hazard function. The additive hazards model has been studied by Lin and Ying (1994). Furthermore, to accommodate both the multiplicative and additive hazards structures, Lin and Ying (1995) proposed a multiplicative-additive hazards model where the hazard function takes the form

$$
\lambda\left(t \mid \mathbf{Z}_{1}(t), \mathbf{Z}_{2}(t)\right)=\lambda(t) \exp \left\{\boldsymbol{\beta}_{1}^{T} \mathbf{Z}_{1}(t)\right\}+\boldsymbol{\beta}_{2}^{T} \mathbf{Z}_{2}(t)
$$

where $\mathbf{Z}_{1}(t)$ and $\mathbf{Z}_{2}(t)$ are different covariates of $\mathbf{Z}(t)$. However, all these hazard-based regression models are restrictive in practice, since they may not be flexible enough to entertain situations where hazard risks are neither multiplicative nor additive among groups. Therefore, it is desirable to obtain a class of hazard-based models that allows a wide range of hazard structures, while at the same time retains the simple structures of the multiplicative and additive hazards models.

This paper proposes a unified family of hazard-based regression models. We propose a class of transformed hazards models by imposing both an additive structure and a known transformation $G(\cdot)$ on the hazard function. In this class, the hazard function for the survival times given covariate $\mathbf{Z}(t)$ takes the form

$$
G\{\lambda(t \mid \mathbf{Z}(t))\}=\mu(t)+\boldsymbol{\beta}^{T} \mathbf{Z}(t)
$$

where $\boldsymbol{\beta}$ is the unknown regression coefficient vector, $\mu(t)$ is an unknown baseline hazard function and $G(\cdot)$ is a known and increasing transformation function. Essentially, model (1) can be 
regarded as a partial linear regression model for the transformed hazard function. One example of the transformation $G(\cdot)$ is the Box-Cox transformation (Box and Cox, 1964) in which $G(x)$ is given by

$$
G(x)=\left(x^{s}-1\right) / s
$$

for $s>0$ and we define $G(x)=\log (x)$ if $s=0$. Within the Box-Cox transformation family, when $s=1$ in (2), (1) is the additive hazards model, and if $s=0$, then (1) becomes the multiplicative hazards model. Thus, the transformed model in (1) with $G(\cdot)$ given by (2) can be considered as a smoothed class of hazards models linking the additive and multiplicative hazards models, which are the extremes of this class if $s$ is restricted to the range of $[0,1]$. Since our proposed class (1) allows a much broader class of hazard patterns than those of the proportional hazards model and the additive hazards model, it provides us with more flexible models for analyzing survival data.

Our goal in this paper is to provide a unified framework in deriving an efficient estimate for $\boldsymbol{\beta}$ in model (1) for any given transformation $G$, where $G^{-1}$ is continuously three times differentiable. In particular, we use the sieve maximum likelihood estimation approach to construct an estimate of $\boldsymbol{\beta}$. We then examine the asymptotic properties of the resulting estimator.

The rest of this article is organized as follows. In Section 2, we present a general framework of sieve maximum likelihood estimation. In Section 3, we derive the asymptotic properties of the estimator, including consistency and asymptotic normality. In Section 4, simulation studies are conducted to examine the numerical properties of the proposed method in small samples. In Section 5, a lung cancer dataset is analyzed using the proposed class of models and estimation procedure. We present a brief discussion in Section 6. Proofs of all theorems are given in the Appendix.

\section{Inference Procedure}

Suppose that we observe survival data with $n$ independent and identically distributed (i.i.d.) observations in a study with termination time $\tau$. We denote the observation for subject $i$ as $\left(Y_{i}=T_{i} \wedge C_{i}, \Delta_{i}=I\left(T_{i} \leq C_{i}\right),\left\{\mathbf{Z}_{i}(t): t \in[0, \tau]\right\}\right)$, where $T_{i}$ is the failure time of subject $i, C_{i}$ is the censoring time, $\left\{\mathbf{Z}_{i}(t): t \in[0, \tau]\right\}$ denotes the external covariate process, " $\wedge$ " denotes the minimum of two values, and $I(\cdot)$ is the indicator function.

We assume that $C_{i}$ is independent of $T_{i}$ conditional on the covariates. Under the assumption 
that the transformation $G(\cdot)$ in the model (1) is strictly increasing and differentiable, the observed likelihood function of the parameters $(\boldsymbol{\beta}, \mu)$ can be written as

$$
L_{n}(\boldsymbol{\beta}, \mu)=\prod_{i=1}^{n}\left\{H\left(\mu\left(Y_{i}\right)+\boldsymbol{\beta}^{T} \mathbf{Z}\left(Y_{i}\right)\right)\right\}^{\Delta_{i}} \exp \left\{-\int_{0}^{Y_{i}} H\left(\mu(t)+\boldsymbol{\beta}^{T} \mathbf{Z}(t)\right) d t\right\},
$$

where $H(\cdot)$ is the inverse function of $G(\cdot)$.

To obtain estimates for $\boldsymbol{\beta}$ and $\mu(t)$, we wish to maximize $L_{n}(\boldsymbol{\beta}, \mu)$ in (3). However, such a maximum does not exist since one can always find some function $\mu(t)$ such that $L_{n}(\boldsymbol{\beta}, \mu)=\infty$. Therefore, we must restrict $\mu(t)$ to some smaller functional space to ensure that the maximum of $L_{n}(\boldsymbol{\beta}, \mu)$ exists. One important method is sieve maximum likelihood estimation, which has been used in many semiparametric estimation problems (Shen and Wong, 1994; Shen, 1997, 1998). In the sieve estimation method, the infinite-dimensional functional parameter $\mu(t)$ is restricted to a functional space with finite dimension, which is called the sieve space for $\mu(t)$. Moreover, the size of this sieve space increases with sample size $n$, and as $n \rightarrow \infty$, the sieve space approximates the whole space for $\mu(t)$. However, for fixed sample size $n$, the choice of the sieve space for $\mu(t)$ cannot be arbitrary: the space should be chosen large enough so that the bias of the sieve estimate for $\mu(t)$ does not dominate; on the other hand, the space cannot be chosen too large so that the variation in estimating $\mu(t)$ dominates the variation in estimating $\boldsymbol{\beta}$, which is the main parameter of interest. Once a sieve space is chosen, maximizing the likelihood function can be carried out on this space, which contains only a finite number of parameters.

Usually, the sieve space for $\mu(t)$ is constructed from a linear space with a finite number of basis functions. Many basis functions can be used for this purpose. The most commonly used basis functions include B-splines and wavelet basis functions. In this paper, we choose wavelet basis functions to construct a sieve space for $\mu(t)$ for reasons of both mathematical and computational convenience, as will be seen in the subsequent arguments. A sequence of wavelet basis functions can be obtained from a single function $\phi(t)$, which is called the father wavelet, and satisfies:

(i) $\{\phi(t-k): k \in \mathcal{Z}\}$ is an orthonormal system in $L_{2}(R)$, where $\mathcal{Z}$ consists of all the integers,

(ii) denote $V_{j}=\left\{\sum_{k} c_{k} \phi\left(2^{j} t-k\right): \sum_{k}\left|c_{k}\right|^{2}<\infty\right\}$ for any $j \geq 0$, then $V_{0} \subset V_{1} \subset \ldots$, and $\cup_{j \geq 0} V_{j}$ is dense in $L_{2}(R)$.

The sequence $\left\{V_{j}: j=0,1, \ldots\right\}$ is called a multi-resolution approximation in the wavelet 
analysis (Section 7.1, Mallat, 1998). From (ii), the basis functions $\left\{\phi\left(2^{j} t-k\right)\right\}$ from $V_{j}$ for some suitable $j$ can be candidates for constructing a sieve space. Furthermore, the orthogonality given in (i) concludes that the $L_{2}$-distance between any two functions in the sieve space can be expressed as the summed square difference of the coefficients of the basis functions, which does not hold for B-spline sieves. We note that $V_{j}$ is still of infinite dimension. However, since our function $\mu(t)$ is of interest only for $t \in[0, \tau]$, the basis functions in $V_{j}$ whose supports do not overlap with $[0, \tau]$ can be discarded. Thus, the number of those remaining basis functions is finite, particularly if we choose $\phi(t)$ to have a compact support. Furthermore, $\phi(t)$ needs to be smooth to ensure the approximation of the sieve space to the whole space for $\mu(t)$. In summary, we assume that the father wavelet $\phi(t)$ satisfies:

(iii) $\phi(t)$ has a finite support $[0, \tau]$ and $\phi \in W^{3,2}[0, \tau]$, where $W^{3,2}[0, \tau]$ is a Sobolev space containing all the functions whose third derivatives are $L_{2}$-integrable in $[0, \tau]$ (c.f. Chapter 1, Adams, 1975).

Typical choices of $\phi(t)$ satisfying (iii) are the Daubechies wavelets (Daubechies, 1992), after suitable shifting and scaling. In the commercial package MATLAB, the Wavelet Toolbox provides a number of these choices.

After $\phi(t)$ is given, we can approximate the function $\mu(t), t \in[0, \tau]$, using the functions in the $K_{n}$-level multi-resolution $V_{K_{n}}$. We choose the basis functions from $\left\{\phi\left(2^{K_{n}} t-k+1\right)\right.$ : $\left.1-\tau \leq k \leq 2^{K_{n}} \tau+1\right\}$ whose supports overlap with $[0, \tau]$. Let $B_{1}(t), \ldots, B_{m_{n}}(t)$ denote these basis functions, where $m_{n}$ is the number of integers between $1-\tau$ and $2^{K_{n}} \tau+1$. Additionally, we impose an upper bound $M_{n}$ for the summation of absolute values of all the wavelet coefficients to prevent the divergence of these coefficients in the maximization. As a result, a sieve space for the parameters $(\boldsymbol{\beta}, \mu)$ is proposed as

$$
\mathcal{S}_{n}=\left\{\begin{array}{c}
(\boldsymbol{\beta}, \mu(t)): \mu(t)=\sum_{k=1}^{m_{n}} \alpha_{k} B_{k}(t), B_{k}(t)=\phi\left(2^{K_{n}} t-k+1\right), \sum_{k=1}^{m_{n}}\left|\alpha_{k}\right| \leq M_{n}, \\
\left.\boldsymbol{\beta} \in \mathcal{B}_{0}, \mathcal{B}_{0} \text { is a known bounded open set containing the true value of } \boldsymbol{\beta}\right\},
\end{array}\right.
$$

where $M_{n}$ is a constant depending on $n$ and the choice of $M_{n}$ is discussed in Section 3 .

We thus maximize the likelihood function $L_{n}(\boldsymbol{\beta}, \mu)$ over $\mathcal{S}_{n}$. The maximization is carried out by an optimum search over the space

$$
\left\{\left(\boldsymbol{\beta}, \alpha_{1}, \ldots, \alpha_{m_{n}}\right): \boldsymbol{\beta} \in \mathcal{B}_{0}, \sum_{k=1}^{m_{n}}\left|\alpha_{k}\right| \leq M_{n}\right\} .
$$


Many optimization algorithms can be implemented to estimate the parameters. Particularly, in the numerical computations of Section 4, we use the algorithm for searching the optimum in MATLAB. The details of the computational procedure are discussed in Section 4.

We denote the sieve maximum likelihood estimate for $(\boldsymbol{\beta}, \mu)$ as $(\hat{\boldsymbol{\beta}}, \hat{\mu})$. Our subsequent results show that $\sqrt{n}\left(\hat{\boldsymbol{\beta}}-\boldsymbol{\beta}_{0}\right)$ has an asymptotically normal distribution with mean zero and covariance matrix $\boldsymbol{\Sigma}$, which is equal to the semiparametric efficiency bound for $\boldsymbol{\beta}$. Unfortunately, $\boldsymbol{\Sigma}$ does not have an explicit expression. Thus, to estimate the asymptotic covariance of $\hat{\boldsymbol{\beta}}$, we propose the following sieve profile likelihood function. We define

$$
p l_{n}(\boldsymbol{\beta})=\max _{\mu \in \mathcal{S}_{n}} \log L_{n}(\boldsymbol{\beta}, \mu)
$$

Then for any constant vector $\mathbf{e}$, we can approximate $\mathbf{e}^{T} \boldsymbol{\Sigma}^{-1} \mathbf{e}$ by

$$
-\frac{1}{n h_{n}^{2}}\left\{p l_{n}\left(\hat{\boldsymbol{\beta}}+h_{n} \mathbf{e}\right)-2 p l_{n}(\hat{\boldsymbol{\beta}})+p l_{n}\left(\hat{\boldsymbol{\beta}}-h_{n} \mathbf{e}\right)\right\}
$$

where $h_{n}$ is a constant of order $1 / \sqrt{n}$. The sieve profile likelihood function imitates the profile likelihood function investigated in Murphy and van der Vaart (2000), and has been discussed by Fan and Wong (2000). Additionally, likelihood ratio inference based on the sieve likelihood function has been recently studied by Shen and Zhang (2004) and Fan and Zhang (2004). Our simulation study in Section 4 shows that for moderate sample sizes, the profile sieve likelihood approach gives valid estimates of the variance.

\section{Asymptotic Properties}

In this section, we obtain the asymptotic properties for $\hat{\boldsymbol{\beta}}$. In particular, we show that the sieve maximum likelihood estimate $(\hat{\boldsymbol{\beta}}, \hat{\mu})$ is consistent under some suitable metric. Next, we show that $\sqrt{n}\left(\hat{\boldsymbol{\beta}}-\boldsymbol{\beta}_{0}\right)$ converges in distribution to a normal distribution and the asymptotic variance attains the semiparametric efficiency bound (c.f., Chapter 3, Bickle, Klaassen, Ritov and Wellner, 1993). All proofs are given in the appendix.

To establish these results, we assume that the following conditions hold:

(C.1) With probability one, $\{\mathbf{Z}(t): t \in[0, \tau]\}$ is a bounded process. Moreover, if there exists some vector $\tilde{\boldsymbol{\beta}}$ such that, $\tilde{\boldsymbol{\beta}}^{T} \mathbf{Z}(t)=c(t)$ for some deterministic function $c(t)$, then $\tilde{\boldsymbol{\beta}}=0$ and $c(t)=0$. 
(C.2) $C$ is independent of $T$ given $\{\mathbf{Z}(t): t \in[0, \tau]\}$. Moreover, with probability one,

$$
\inf _{\mathbf{z}(t), t \in[0, \tau]} P(C \geq \tau \mid \mathbf{Z}(t)=\mathbf{z}(t), t \in[0, \tau])=\inf _{\mathbf{z}(t), t \in[0, \tau]} P(C=\tau \mid \mathbf{Z}(t)=\mathbf{z}(t), t \in[0, \tau])>0
$$

(C.3) Denote the true values of $(\boldsymbol{\beta}, \mu)$ by $\left(\boldsymbol{\beta}_{0}, \mu_{0}\right)$. Assume that $\boldsymbol{\beta}_{0} \in \mathcal{B}_{0}$ and $\mu_{0}(t)$ is a continuously three times differentiable function in $[0, \tau]$. Moreover, assume

$$
\inf _{t \in[0, \tau]} H\left(\mu_{0}(t)+\boldsymbol{\beta}_{0}^{T} \mathbf{Z}(t)\right)>0, \quad \sup _{t \in[0, \tau]}\left|\mu_{0}^{\prime \prime \prime}(t)\right|<\infty .
$$

Assumption (C.1) ensures the identifiability of $\boldsymbol{\beta}$ in model (1). (C.2) implies that the distribution for the censoring times is not informative, and therefore $L_{n}(\boldsymbol{\beta}, \mu)$ is the only part in the full likelihood function we need to maximize. The second part of (C.2) is equivalent to saying that any subjects surviving to at least $\tau$ are considered as right-censored at $\tau$. Both (C.1) and (C.2) are standard assumptions in the Cox proportional hazards model. Assumption (C.3) implies that the true conditional hazard rate for $T$ given the covariates is bounded away from zero.

We also need assumptions for the choices of $m_{n}$ (or $K_{n}$ ) and $M_{n}$. Specifically, we assume that the number of basis functions in the sieve space increases with sample size $n$ but at a low rate. Moreover, we assume that the upper bound $M_{n}$ in the sieve space should tend toward infinity at an appropriate rate depending on the transformation function $H$. The details are given in the following theorem.

Theorem 1. In addition to conditions (C.1)-(C.3), for each $M_{n}>0$, define $\gamma_{1}\left(M_{n}\right)=2 H\left(M_{n}+B\right), \gamma_{2}\left(M_{n}\right)=\sup _{x \in\left[-M_{n}-B, M_{n}+B\right]} H^{\prime}(x), \gamma_{3}\left(M_{n}\right)=\left\{\inf _{x \in\left[-M_{n}-B, M_{n}+B\right]} H^{\prime}(x)\right\}^{-1}$, where $B$ is the upper bound of $\left|\boldsymbol{\beta}^{T} \mathbf{Z}(t)\right|$. Assume that

(C.4) $m_{n}$ satisfies that $m_{n} \rightarrow \infty$ and $m_{n}^{7} / n \rightarrow 0$. Moreover, $M_{n}$ satisfies

$$
M_{n}^{1 / 3} \xi\left(M_{n}\right)^{2 / 3}\left(\sqrt{\frac{m_{n}^{7 / 6}}{n^{1 / 6}}}+\frac{1}{m_{n}}\right) \rightarrow 0
$$

where $\xi\left(M_{n}\right)=M_{n}^{2} \gamma_{1}\left(M_{n}\right)^{2} \gamma_{2}\left(M_{n}\right)^{4} \gamma_{3}\left(M_{n}\right)^{2}$.

Then $\hat{\boldsymbol{\beta}}$ and $\hat{\mu}(\cdot)$ are consistent in the sense that $\left|\hat{\boldsymbol{\beta}}-\boldsymbol{\beta}_{0}\right|+\left\|\hat{\mu}-\mu_{0}\right\|_{L_{2}[0, \tau]} \rightarrow 0$ in probability. 
The first part of (C.4) stipulates that the number of basis functions in the sieve space, $m_{n}$, increases at a lower rate than $n^{1 / 7}$. We also remark that $M_{n}$ satisfying (C.4) always exists for a given $m_{n}$ and $n$. Some particular choices of $m_{n}$ and $M_{n}$ for the class of Box-Cox transformations are given at the end of this section. The convergence rates of $(\hat{\boldsymbol{\beta}}, \hat{\mu})$ are obtained explicitly in the following theorem.

Theorem 2. Under conditions (C.1)-(C.4),

$$
\int_{0}^{\tau}\left\{\left(\hat{\mu}(t)-\mu_{0}(t)\right)+\left(\hat{\boldsymbol{\beta}}-\boldsymbol{\beta}_{0}\right)^{T} \mathbf{Z}(t)\right\}^{2} d t \leq o_{p}\left(\frac{1}{\sqrt{n}}\right)+O_{p}\left(\frac{1}{m_{n}^{6}}\right) .
$$

Finally, the asymptotic distribution for $\hat{\boldsymbol{\beta}}$ can be summarized in the following theorem.

Theorem 3. In addition to conditions (C.1)-(C.4), suppose that with probability one, $\mathbf{Z}(t)$ is continuously three times differentiable in $[0, \tau]$, and with respect to some dominating measure, the conditional density of $C$ given $\{\mathbf{Z}(t): t \in[0, \tau]\}$ is three times continuously differentiable. Moreover, $H=G^{-1}$ is continuously three times differentiable and $m_{n}$ satisfies

(C.5) $\sqrt{n} / m_{n}^{6} \rightarrow 0$

Then $\sqrt{n}\left(\hat{\boldsymbol{\beta}}-\boldsymbol{\beta}_{0}\right)$ converges weakly to a normal distribution with mean zero and its asymptotic variance attains the semiparametric efficiency bound.

The regularity condition for $\mathbf{Z}(t)$ in Theorem 3 holds when $\mathbf{Z}(t)$ is time-independent. Since from Theorem 2, the bias of the sieve estimate $\hat{\mu}(t)$ is of the order $m_{n}^{-12}$, assumption (C.5) implies that the square of this bias does not dominate the variation of $\hat{\boldsymbol{\beta}}$, which is of the order $n^{-1 / 2}$.

The choices of $K_{n}$ and $M_{n}$ satisfying (C.4) and (C.5) exist. For large $n$, we can choose $K_{n}=\theta \log n / \log 2\left(\operatorname{thus} m_{n}=n^{\theta}\right)$, where $\theta$ is a constant in the interval $(1 / 12,1 / 7)$. If $G$ is the Box-Cox transformation, $M_{n}$ can be particularly chosen to be of the order $\log n$.

\section{Simulation Study}

We conducted simulation studies to examine the small-sample performance of our proposed estimators. In the simulation, we generated two independent covariates $Z_{1}$ and $Z_{2}$ from 
Uniform $(0,1)$ and Bernoulli(0.5), respectively. The failure time was generated from the following model:

$$
\frac{\left\{\lambda\left(t \mid Z_{1}, Z_{2}\right)\right\}^{s}-1}{s}=\frac{t}{2}+\beta_{1} Z_{1}+\beta_{2} Z_{2}
$$

where $\beta_{1}=0.7$ and $\beta_{2}=0.2$. We varied the choices of $s$ using the values of $0,0.25,0.5,0.75$ and 1 . Thus, when $s=0$, the failure time was generated from a proportional hazards regression model with baseline hazard, $\exp (t / 2)$; when $s=1$, the failure time was from an additive hazards model with baseline hazard, $t / 2+1$. The censoring time was taken as the minimum of 1 and $C^{*}$ where $C^{*} \sim$ Uniform $(0.5,1.5)$ and the censoring rates varied from $20 \%$ to $25 \%$ for $s=0$ to $s=1$.

For each $s$, we simulated 500 data sets, and for each data realization, the proposed sieve maximum likelihood estimation approach was used to estimate the regression coefficients. In sieve estimation, we chose the Db4-father wavelet (Daubechies, 1992) for $\phi(t)$ and used resolution level $K_{n}=3$ to estimate the nuisance parameter $\mu(t)$. The sieve maximum likelihood estimates were obtained by the algorithm for searching the optimum in the Optimization Toolbox in MATLAB. This algorithm is a subspace trust region method and is based on the interior-reflective Newton method (Coleman and Li, 1994; 1996), after both gradients and Hessian derivatives of the objective function are provided. Since the objective function may not be concave in the parameters, choosing initial values can be very important. In our experience, when the initial values were chosen not too far away from the true values, the estimates at convergence were very similar. In the simulation study, the optimum search usually converged within a few iterations when either the step size of the search or the gradient of the function was very small. The sieve profile likelihood function was used to estimate the asymptotic variance of $\hat{\boldsymbol{\beta}}$, where we chose $h_{n}=n^{-1 / 2}$. In the simulation study, we also used $K_{n}=4,5$ and $h_{n}=0.1 n^{-1 / 2}, 5 n^{-1 / 2}$ and found the results to be fairly robust with respect to these choices.

Table 1 summarizes the simulation results for different choices of $s$ values for $n=200$ and $n=400$, respectively. The columns after the true value correspond to the average values of the estimates, the standard errors of the estimates, the average estimates of the asymptotic standard errors, and the coverage proportions of the $95 \%$ confidence intervals, based on the normal distribution. The results in Table 1 indicate that the sieve maximum likelihood estimates for the regression coefficients have a small bias; the estimated standard errors based on the sieve profile likelihood function are close to the empirical standard errors; and the coverage proportions of $95 \%$ confidence intervals are accurate. Increasing the sample size from 200 to 
400 decreases both the bias and the standard errors of the estimates.

\section{Application}

The proposed approach was applied to a lung cancer dataset from a recent phase III clinical trial (Socinski et al., 2002) of non-small-cell lung cancer (NSCLC), which is the leading cause of cancer-related mortality. In the year 2001, among approximately 170,000 patients newly diagnosed, more than 90\% died from NSCLC and approximately $35 \%$ of all new cases were stage IIIB/IV (malignant pleural effusion) the disease. A randomized, two-armed, multi-center trial was initiated in 1998 with the aim to determine the optimal duration of chemotherapy by comparing four cycles of therapy versus continuous therapy in advanced NSCLC. Patients were randomized to two treatment arms: four cycles of carboplatin at an area under the curve of 6 and paclitaxel $200 \mathrm{mg} / \mathrm{m}^{2}$ every 21 days (arm A), or continuous treatment with carboplatin/paclitaxel until progression (arm B). At progression, all patients on both arms received second-line weekly paclitaxel at $80 \mathrm{mg} / \mathrm{m}^{2}$. One of the primary endpoints was survival, which could be right-censored due to loss to follow-up. The original dataset had 230 NSCLC patients, and 4 cases were missing follow-up times, and hence our analysis is based on $n=226$ cases, of which 113 were in arm A and 113 were in arm B. The censoring rate was approximately $32 \%$.

We illustrate the proposed additive transformation hazards models with these NSCLC data and demonstrate the flexibility and generality of this class of models. The covariates included in the model were treatment $(0=\operatorname{arm~} A, 1=\operatorname{arm} B)$, sex $(0=$ female, $1=$ male $)$ and age at entry. In this population, $63 \%$ of the patients were male, and the age at entry ranged from 32 to 82 with a mean of 62 years. In the analysis, we re-scaled the time axis to the interval $[0,1]$.

We fit a class of Box-Cox transformed hazard models to the NSCLC data. The parameter $s$ in the transformation was chosen as $0,0.25,0.5,0.75$ and 1 ; the multi-resolution level $K_{n}$ was chosen from 2, 3, 4 and 5. The Akaike information criteria (AIC), defined as twice the negative log-likelihood function plus twice the number of the parameters, was used as a criterion to select the best fitting model. Using the AIC by varying $s$ and $K_{n}$ ensured the best model choice in terms of both model structure and parsimony, although it is difficult to distinguish whether the best fit is due to the transformation or the choice of basis functions. We also penalized those choices of $s$ and $K_{n}$ for which the estimated parameters induced negative predicted values for the hazard function. If the estimated hazard rate was negative, we set the objective function that needed to be maximized, to be a very small negative number. Thus, the best model using AIC 
always ensures that the predicted hazard function is positive. From the analysis, we found that increasing the number of basis functions dramatically increased the value of AIC and the model with $s=0.5$ and $K_{n}=2$ yielded the minimal AIC value. The estimates and standard errors for

the coefficients of the three covariates are: $\hat{\beta}_{\text {treat }}=-0.1176(0.2841), \hat{\beta}_{\text {sex }}=0.7086(0.2966)$, and $\hat{\beta}_{\text {age }}=0.6568(0.5332)$. Thus, only the covariate sex is significantly predictive of hazard risk. The male patients had a higher risk than the females. Neither treatment nor age is significant. We also plotted the predicted survival curves versus the Kaplan-Meier survival curves in Figure 1. Each plot in Figure 1 represents the predicted survival curves and the Kaplan-Meier curves stratified by treatment and sex, where the age value is substituted with its median value 63 . The plots indicate that the best model $(s=0.5, K=2)$ indeed provides a good fit to the data.

\section{Discussion}

We have proposed a class of transformation models for modelling the hazard function. This class of models contains both multiplicative and additive hazards models as special cases. A unified estimation procedure has been proposed, where the sieve maximum likelihood estimates were obtained by maximizing the observed likelihood function over a sieve space of wavelets. The resulting estimators for the regression coefficients have been shown to be asymptotically normal. Simulation studies indicated that the proposed estimates performed well for sample sizes of 200 and 400. Applying the Box-Cox transformed hazards model to the lung cancer data demonstrated that the best model might not be either the multiplicative or additive hazards model.

In the optimization for computing the maximum likelihood estimates, choosing initial values is an important issue. Although our numerical studies indicate that if initial values are not far from true values, convergence is often satisfactory, one has to guess an initial value in practice. One possible way is to use the estimates from the proportional hazards model, which corresponds to transformation $H(x)=\exp \{x\}$ and which has the concave log-likelihood function, as the initial values. Another more general solution is to choose a few widespread points in the parameter domain as initial values and among all the estimates starting from these initial values, the one with the maximal likelihood function is considered to be the maximum likelihood estimate.

Although in our theoretical derivations, a high-order smooth father wavelet is required to ensure that the asymptotic results hold for the regression parameters, our simulation study and 
data application showed that using a low-order smooth father wavelet (e.g., the Db4 wavelet) works quite well. In practice, if one is only interested in inference on the regression coefficients, a low-order smooth wavelet basis such as the Db4 wavelet may be used; while a high-order smooth wavelet should be used if one is interested in obtaining a smooth predicted function of the hazard rate.

In many other nonparametric estimation contexts, it is important to choose a suitable smoothing parameter. In the sieve maximum likelihood estimation we have proposed, such a parameter is the multi-resolution level $K_{n}$ (thus $m_{n}$ ). In the data application, we have used the AIC criterion to choose $K_{n}$. In fact, some other criteria can be used in choosing $K_{n}$ : one possibility is to replace the negative log-likelihood function in the AIC criterion by a distance measure, which is defined as the $L_{2}$-distance between the predicted survival function based on the model and the Kaplan-Meier survival function. The AIC criterion or the criterion proposed above can also be used to choose the model that best fits the data from a class of transformed hazards models, as we did in the data application. In all these model selection procedures, the variation in choosing the best model is not accounted for in our inference for the regression parameters. One possibility for accounting for such variation is to treat the transformation $G$, indexed by the parameter $s$, as another model parameter; then we maximize the observed likelihood function over all model parameters including the transformation $G$. However, the asymptotic properties of the estimators for the regression coefficients are not yet available.

\section{APPENDIX}

\section{A.1. Proof of Theorem 1}

The consistency proof contains the following steps, where $r=3$.

Step 1. We first choose $\tilde{\mu}(t)$ as the approximate function in the $K_{n}$ th multi-resolution to $\mu_{0}(t)$ such that $\left(\boldsymbol{\beta}_{0}, \tilde{\mu}\right) \in \mathcal{S}_{n}$. According to the results in the wavelet analysis (Section 9.4, Härdle, Kerkyacharian, Picard and Tsybakov, 2000),

$$
\left\|\tilde{\mu}-\mu_{0}\right\|_{W^{1, \infty}} \leq O(1) \frac{\left\|\mu_{0}\right\|_{W^{r, \infty}}}{m_{n}^{r-1}}, \quad\left\|\tilde{\mu}-\mu_{0}\right\|_{L^{\infty}} \leq O(1) \frac{\left\|\mu_{0}\right\|_{W^{r, \infty}}}{m_{n}^{r}}
$$

where $\|\mu\|_{W^{r, \infty}}=\sup _{l \leq r} \sup _{t \in[0, \tau]}\left|\mu^{(j)}(t)\right|$ for $k=0, \ldots, r$. Moreover, the wavelet coefficients for $\tilde{\mu}(t)=\sum_{j=1}^{m_{n}} \tilde{\alpha}_{j} B_{j}(t)$ satisfy that $\sum_{j=1}^{m_{n}}\left|\tilde{\alpha}_{j}\right|<\infty$. Thus, $\left(\boldsymbol{\beta}_{0}, \tilde{\mu}\right) \in \mathcal{S}_{n}$. 
Step 2. We obtain a bound for the distance

$$
d\left((\hat{\boldsymbol{\beta}}, \hat{\mu}),\left(\boldsymbol{\beta}_{0}, \mu_{0}\right)\right) \equiv\left|\hat{\boldsymbol{\beta}}-\boldsymbol{\beta}_{0}\right|+\left\|\hat{\mu}-\mu_{0}\right\|_{L_{2}}
$$

where $\|\mu\|_{L_{2}}=\left\{\int_{0}^{\tau}|\mu(t)|^{2} d t\right\}^{1 / 2}$. From the construction of $\tilde{\mu}$, we immediately obtain that

$$
L_{n}(\hat{\boldsymbol{\beta}}, \hat{\mu}) \geq L_{n}\left(\boldsymbol{\beta}_{0}, \tilde{\mu}\right) .
$$

If we denote $\mathbf{P}_{n}$ as the empirical measure based on the $n$ i.i.d observations and denote $\mathbf{P}$ as the corresponding expectation, then after taking the log on both sides of (4) and dividing by $n$, we have that

$$
\begin{aligned}
& \mathbf{P}_{n}\left\{\Delta \log H\left(\hat{\mu}(Y)+\hat{\boldsymbol{\beta}}^{T} \mathbf{Z}(Y)\right)-\int_{0}^{Y} H\left(\hat{\mu}(t)+\hat{\boldsymbol{\beta}}^{T} \mathbf{Z}(t)\right) d t\right\} \\
\geq & \mathbf{P}_{n}\left\{\Delta \log H\left(\tilde{\mu}(Y)+\boldsymbol{\beta}_{0}^{T} \mathbf{Z}(Y)-\int_{0}^{Y} H\left(\tilde{\mu}(t)+\boldsymbol{\beta}_{0}^{T} \mathbf{Z}(t)\right) d t\right\} .\right.
\end{aligned}
$$

Note that the function $\Delta \log H(\cdot)-\int_{0}^{Y} H(\cdot) d t$ is concave in $H(\cdot)$. Thus, for any $\delta_{n}>0$, if we define

$$
H_{\delta_{n}}(t)=\delta_{n} H\left(\hat{\mu}(t)+\hat{\boldsymbol{\beta}}^{T} \mathbf{Z}(t)\right)+\left(1-\delta_{n}\right) H\left(\tilde{\mu}(t)+\boldsymbol{\beta}_{0}^{T} \mathbf{Z}(t)\right),
$$

then we have

$\mathbf{P}_{n}\left\{\Delta \log H_{\delta_{n}}(Y)-\int_{0}^{Y} H_{\delta_{n}}(t) d t\right\} \geq \mathbf{P}_{n}\left\{\Delta \log H\left(\tilde{\mu}(Y)+\boldsymbol{\beta}_{0}^{T} \mathbf{Z}(Y)\right)-\int_{0}^{Y} H\left(\tilde{\mu}(t)+\boldsymbol{\beta}_{0}^{T} \mathbf{Z}(t)\right) d t\right\}$.

Thus,

$$
\begin{aligned}
& n^{-1 / 2} \mathbf{G}_{n}\left\{\Delta \log H_{\delta_{n}}(Y)-\int_{0}^{Y} H_{\delta_{n}}(t) d t-\Delta \log H\left(\tilde{\mu}(Y)+\boldsymbol{\beta}_{0}^{T} \mathbf{Z}(Y)\right)+\int_{0}^{Y} H\left(\tilde{\mu}(t)+\boldsymbol{\beta}_{0}^{T} \mathbf{Z}(t)\right) d t\right\} \\
& \geq-\mathbf{P}\left\{\Delta \log H_{\delta_{n}}(Y)-\int_{0}^{Y} H_{\delta_{n}}(t) d t-\Delta \log H\left(\tilde{\mu}(Y)+\boldsymbol{\beta}_{0}^{T} \mathbf{Z}(Y)\right)+\int_{0}^{Y} H\left(\tilde{\mu}(t)+\boldsymbol{\beta}_{0}^{T} \mathbf{Z}(t)\right) d t\right\},
\end{aligned}
$$

where $\mathbf{G}_{n}$ denotes the empirical process $\sqrt{n}\left(\mathbf{P}_{n}-\mathbf{P}\right)$.

We now want to bound the left-hand side of (5) using the results of the empirical process theory. Towards this goal, we choose $\delta_{n}$ such that for some small constant $\delta_{0}, \delta_{n}=$ $\delta_{0} /\left\{M_{n} \gamma_{1}\left(M_{n}\right) \gamma_{2}\left(M_{n}\right)\right\}$, where $\gamma_{1}\left(M_{n}\right)=2 H\left(M_{n}+B\right)$ and $\gamma_{2}\left(M_{n}\right)=\sup _{x \in\left[-M_{n}-B, M_{n}+B\right]} H^{\prime}(x)$. Hence,

$$
\left\|H_{\epsilon}(t)-H\left(\tilde{\mu}(t)+\boldsymbol{\beta}_{0}^{T} \mathbf{Z}(t)\right)\right\|_{L_{\infty}} \leq \delta_{n}\left\|H\left(\hat{\mu}(t)+\hat{\boldsymbol{\beta}}^{T} \mathbf{Z}(t)\right)-H\left(\tilde{\mu}(t)+\boldsymbol{\beta}_{0}^{T} \mathbf{Z}(t)\right)\right\|_{L_{\infty}} \leq \delta_{0} .
$$


Moreover, we define a class of functions

$$
\mathcal{H}_{n}=\left\{\delta_{n} H\left(\mu(t)+\boldsymbol{\beta}^{T} \mathbf{Z}(t)\right)+\left(1-\delta_{n}\right) H\left(\tilde{\mu}(t)+\boldsymbol{\beta}_{0}^{T} \mathbf{Z}(t)\right):(\boldsymbol{\beta}, \mu) \in \mathcal{S}_{n}\right\} .
$$

By the property of the father wavelet, for any $(\boldsymbol{\beta}, \mu) \in \mathcal{S}_{n}$,

$$
\left|\mu^{\prime}(t)\right| \leq \sum_{j=1}^{m_{n}}\left|\alpha_{j}\right|\left|B_{j}^{\prime}(t)\right| \leq c_{0} m_{n} M_{n}
$$

for some constant $c_{0}$ so the $\epsilon$-bracket covering number for the class of such $\mu$ with respect to $L_{2}(P)$-norm is of the order $\exp \left\{O\left(M_{n} m_{n} / \epsilon\right)\right\}$ (Corollary 2.7.2, van der Vaart and Wellner, 1996). By the monotonicity of $H(\cdot)$, we thus can construct the $\exp \left\{O\left(M_{n} m_{n} / \epsilon\right)\right\}$ brackets to cover $\mathcal{H}_{n}$ such that within each bracket, any two functions indexed by $\left(\boldsymbol{\beta}_{1}, \mu_{1}\right)$ and $\left(\boldsymbol{\beta}_{2}, \mu_{2}\right)$ respectively satisfy $\left|\boldsymbol{\beta}_{1}-\boldsymbol{\beta}_{2}\right|+\left\|\mu_{1}-\mu_{2}\right\|_{L_{2}(P)} \leq \epsilon$. However, since

$$
\left.\delta_{n} H^{\prime}(x)\right|_{x=\mu(t)+\boldsymbol{\beta}^{T} \mathbf{z}(t)} \leq \delta_{n} O\left(\gamma_{2}\left(M_{n}\right)\right) \leq \frac{O(1)}{M_{n}},
$$

for these two functions,

$$
\left\|\delta_{n} H\left(\mu_{1}(t)+\boldsymbol{\beta}_{1}^{T} \mathbf{Z}(t)\right)-\delta_{n} H\left(\mu_{2}(t)+\boldsymbol{\beta}_{2}^{T} \mathbf{Z}(t)\right)\right\|_{L_{2}(P)} \leq O\left(\epsilon / M_{n}\right)
$$

We thus conclude that

$$
N_{[]}\left(\epsilon, \mathcal{H}_{n}, L_{2}(P)\right) \leq O\left(\exp \left\{O\left(m_{n} / \epsilon\right)\right\}\right) .
$$

Consequently, another class of functions, which is defined as

$$
\mathcal{F}_{n}=\left\{\Delta \log H_{\delta_{n}}(Y)-\int_{0}^{Y} H_{\delta_{n}}(t) d t: H_{\delta_{n}} \in \mathcal{H}_{n}\right\}
$$

has the bracket covering number of the order

$$
N_{[]}\left(\epsilon, \mathcal{F}_{n}, L_{2}(P)\right) \leq O\left(\exp \left\{O\left(m_{n} / \epsilon\right)\right\}\right)
$$

Note that $\mathcal{F}_{n}$ has a bounded covering function. According to Lemma 19.38, van der Vaart (1998), we obtain that

$$
E_{p}^{*}\left\|\mathbf{G}_{n}\right\|_{\mathcal{F}_{n}} \leq \int_{0}^{O(1)} \sqrt{\log N_{[]}\left(\epsilon, \mathcal{F}_{n}, L_{2}(P)\right)} d \epsilon \leq O\left(\sqrt{m_{n}}\right)
$$

This implies that the left-hand side of $(5)$ is bounded by $O_{p}\left(\sqrt{m_{n}} / \sqrt{n}\right)$.

On the other hand, the right-hand side of (5) can be written as

$$
-\mathbf{P}\left\{\Delta \log H_{\delta_{n}}(Y)-\Delta \log H\left(\mu_{0}(Y)+\boldsymbol{\beta}_{0}^{T} \mathbf{Z}(Y)\right)-\int_{0}^{Y} H_{\delta_{n}}(t) d t+\int_{0}^{Y} H\left(\mu_{0}(t)+\boldsymbol{\beta}_{0}^{T} \mathbf{Z}(t)\right) d t\right\}
$$




$$
\begin{gathered}
-\mathbf{P}\left\{\Delta \log H\left(\mu_{0}(Y)+\boldsymbol{\beta}_{0}^{T} \mathbf{Z}(Y)\right)-\Delta \log H\left(\mu_{0}(Y)+\boldsymbol{\beta}_{0}^{T} \mathbf{Z}(Y)\right)\right. \\
\left.-\int_{0}^{Y} H\left(\mu_{0}(t)+\boldsymbol{\beta}_{0}^{T} \mathbf{Z}(t)\right) d t+\int_{0}^{Y} H\left(\tilde{\mu}(t)+\boldsymbol{\beta}_{0}^{T} \mathbf{Z}(t)\right) d t\right\} .
\end{gathered}
$$

We denote the two terms in (6) as $(I)$ and $(I I)$ and denote $H_{0}(Y)$ as $H\left(\mu_{0}(Y)+\boldsymbol{\beta}_{0}^{T} \mathbf{Z}(t)\right)$. Then applying the mean value theorem to the term $(I)$ yields

$$
\begin{aligned}
(I)= & -\mathbf{P}\left\{\frac{\Delta}{H_{0}(Y)}\left(H_{\delta_{n}}(Y)-H_{0}(Y)\right)-\int_{0}^{Y}\left(H_{\delta_{n}}(t)-H_{0}(t)\right) d t\right\} \\
& +\mathbf{P}\left\{\frac{\Delta}{\tilde{H}(Y)^{2}}\left(H_{\delta_{n}}(Y)-H_{0}(Y)\right)^{2}\right\},
\end{aligned}
$$

where $\tilde{H}$ is a function between $H_{0}$ and $H_{\delta_{n}}$. Since $\left(\boldsymbol{\beta}_{0}, \mu_{0}\right)$ maximizes $\mathbf{P}\left\{\Delta \log H\left(\mu(Y)+\boldsymbol{\beta}^{T} \mathbf{Z}(Y)\right)\right.$ $\left.-\int_{0}^{Y} H\left(\mu(t)+\boldsymbol{\beta}^{T} \mathbf{Z}(t)\right) d t\right\}$, the derivative of the previous function along the submodel $\boldsymbol{\beta}=$ $\boldsymbol{\beta}_{0}, \mu(t)=\mu_{0}(t)+\epsilon q(t), \epsilon \in\left(0, \epsilon_{0}\right)$, where $\epsilon_{0}$ is a small positive constant and $q(t)$ is any measurable function in $L_{2}(P)$, should be zero. This gives that

$$
\mathbf{P}\left\{\frac{\Delta}{H_{0}(Y)} H^{\prime}\left(\mu_{0}(Y)+\boldsymbol{\beta}_{0}^{T} \mathbf{Z}(Y)\right) q(Y)-\int_{0}^{Y} H^{\prime}\left(\mu_{0}(t)+\boldsymbol{\beta}_{0}^{T} \mathbf{Z}(t)\right) q(t) d t\right\}=0 .
$$

Thus, the first part of the right-hand side in $(I)$ is zero. Since $\tilde{H}(Y)$ is smaller than some constant and $H^{\prime}(x) \geq 1 / \gamma_{3}\left(M_{n}\right)$ for $x \in\left[-M_{n}-B, M_{n}+B\right]$, we have that

$$
\begin{aligned}
(I) & \geq O(1) \mathbf{P}\left\{\frac{\Delta}{\tilde{H}(Y)^{2}}\left(H_{\delta_{n}}(Y)-H_{0}(Y)\right)^{2}\right\} \\
& \geq O(1) \frac{\delta_{n}^{2}}{\gamma_{2}\left(M_{n}\right)^{2}} E\left[\left\{\left(\hat{\mu}(Y)-\mu_{0}(Y)\right)+\left(\hat{\boldsymbol{\beta}}-\boldsymbol{\beta}_{0}\right)^{T} \mathbf{Z}(Y)\right\}^{2}\right]-O\left(\gamma_{3}\left(M_{n}\right)^{2}\left\|\tilde{\mu}(t)-\mu_{0}\right\|_{L_{2}}^{2}\right) .
\end{aligned}
$$

Similarly, we apply the expansion to the second term $(I I)$ of (6) around the true parameter $\left(\boldsymbol{\beta}_{0}, \mu_{0}\right)$. The fist order in the expansion vanishes and the second order is bounded by $O(1) \int_{0}^{\tau}\left(\tilde{\mu}(t)-\mu_{0}(t)\right)^{2} d t \leq O\left(1 / m_{n}^{2 r}\right)$ from the construction of $\tilde{\mu}(t)$. Thus, the term $(I I)$ is at least $-c_{0} / m_{n}^{2 r}$ for some positive constant $c_{0}$.

Hence, we obtain that

$$
\begin{aligned}
& E\left[\left\{\left(\hat{\mu}(Y)-\mu_{0}(Y)\right)+\left(\hat{\boldsymbol{\beta}}-\boldsymbol{\beta}_{0}\right)^{T} \mathbf{Z}(Y)\right\}^{2}\right] \\
\leq & O(1)\left\{\frac{M_{n}^{2} \gamma_{1}\left(M_{n}\right)^{2} \gamma_{2}\left(M_{n}\right)^{4} \sqrt{m_{n}}}{\sqrt{n}}+\frac{\gamma_{1}\left(M_{n}\right)^{2} \gamma_{2}\left(M_{n}\right)^{4} \gamma_{3}\left(M_{n}\right)^{2}}{m_{n}^{2 r}}\right\} \\
\leq & O(1) \xi\left(M_{n}\right)^{2}\left\{\frac{\sqrt{m_{n}}}{\sqrt{n}}+\frac{1}{m_{n}^{2 r}}\right\} .
\end{aligned}
$$

Since $\{\mathbf{Z}(t): t \in[0, \tau]\}$ is external and linearly independent with the constant, we obtain that

$$
E\left\{\left(\hat{\mu}(Y)-\mu_{0}(Y)\right)^{2}\right\}+\left(\hat{\boldsymbol{\beta}}-\boldsymbol{\beta}_{0}\right)^{T} E\left\{\mathbf{Z}(Y) \mathbf{Z}(Y)^{T}\right\}\left(\hat{\boldsymbol{\beta}}-\boldsymbol{\beta}_{0}\right) \leq O(1) \xi\left(M_{n}\right)^{2}\left(\frac{\sqrt{m_{n}}}{\sqrt{n}}+\frac{1}{m_{n}^{2 r}}\right) .
$$


Furthermore, by assumption (C.4), $E\left\{\mathbf{Z}(Y)^{T} \mathbf{Z}(Y)\right\}>0$. Then it follows that

$$
\int_{0}^{\tau}\left(\hat{\mu}(t)-\mu_{0}(t)\right)^{2} d t+\left|\hat{\boldsymbol{\beta}}-\boldsymbol{\beta}_{0}\right|^{2} \leq O(1) \xi\left(M_{n}\right)^{2}\left(\frac{\sqrt{m_{n}}}{\sqrt{n}}+\frac{1}{m_{n}^{2 r}}\right) .
$$

Thus, by the choices of $M_{n}$ and $K_{n}$ in (C.4), Theorem 1 holds.

\section{A.2. Proof of Theorem 2}

To prove Theorem 2, we need a consistency result of $\hat{\mu}$ under a stronger norm than the $L_{2}$-norm. First, from the construction of $\mathcal{S}_{n}$,

$$
\left\|\hat{\mu}-\mu_{0}\right\|_{W^{r, 2}} \leq O\left(\sum_{j=1}^{m_{n}}\left|\hat{\alpha}_{j}\right|\left\|B_{j}(t)\right\|_{W^{r, 2}}\right) \leq O\left(M_{n}\right) m_{n}^{r}
$$

Then according to the Sobolev interpolation theorem (Adams, 1975), it holds that

$$
\left\|\hat{\mu}^{\prime}(t)-\mu_{0}^{\prime}(t)\right\|_{L_{2}} \leq c_{1}\left\|\hat{\mu}-\mu_{0}\right\|_{W^{r, 2}}^{1 / r}\left\|\hat{\mu}-\mu_{0}\right\|_{L_{2}}^{1-1 / r}
$$

for some constant $c_{1}$. Then,

$$
\begin{aligned}
\left\|\hat{\mu}^{\prime}(t)-\mu_{0}^{\prime}(t)\right\|_{L_{2}} & \leq O(1) m_{n} M_{n}^{1 / r}\left[\xi\left(M_{n}\right)\left\{\frac{m_{n}^{1 / 4}}{n^{1 / 4}}+\frac{1}{m_{n}^{r}}\right\}\right]^{1-1 / r} \\
& \leq O(1) M_{n}^{1 / r} \xi\left(M_{n}\right)^{1-1 / r}\left[\frac{m_{n}^{5 / 4-1 / 4 r}}{n^{1 / 4-1 / 4 r}}+\frac{1}{m_{n}^{r-2}}\right]
\end{aligned}
$$

Based on the choice of $M_{n}$ and $K_{n}$ in (C.4), this term converges to zero. We thus conclude that in probability,

$$
\left|\hat{\boldsymbol{\beta}}-\boldsymbol{\beta}_{0}\right| \rightarrow 0, \quad\left\|\hat{\mu}-\mu_{0}\right\|_{W^{1,2}} \rightarrow 0 .
$$

Additionally, from the Sobolev embedding theorem (Adams, 1975), we have that in probability,

$$
\left\|\hat{\mu}-\mu_{0}\right\|_{L_{\infty}} \rightarrow 0
$$

We further improve the convergence rate of $\hat{\boldsymbol{\beta}}$ and $\hat{\mu}$. We simply repeat Step 2 in proving Theorem 1 and obtain a similar inequality as (5) but $\delta_{n}$ is set to 1 . Then, the left-hand side of (5) belongs to the process $n^{-1 / 2} \mathbf{G}_{n}\left(\mathcal{F}_{n}^{*}\right)$, where

$$
\begin{aligned}
\mathcal{F}_{n}^{*}= & \left\{\Delta \log H\left(\mu(Y)+\boldsymbol{\beta}^{T} \mathbf{Z}(Y)\right)-\Delta \log H\left(\tilde{\mu}(Y)+\boldsymbol{\beta}_{0}^{T} \mathbf{Z}(Y)\right)\right. \\
& \left.-\int_{0}^{Y} H\left(\mu(t)+\boldsymbol{\beta}^{T} \mathbf{Z}(t)\right) d t+\int_{0}^{Y} H\left(\tilde{\mu}(t)+\boldsymbol{\beta}_{0}^{T} \mathbf{Z}(t)\right) d t:\left|\boldsymbol{\beta}-\boldsymbol{\beta}_{0}\right|<\epsilon,\left\|\mu-\mu_{0}\right\|_{W^{1,2}}<\epsilon\right\}
\end{aligned}
$$


for any small number $\epsilon$. Hence, $\mathcal{F}_{n}$ is P-Donsker; thus, the left-hand side is bounded by $o_{p}\left(n^{-1 / 2}\right)$. We again apply a Taylor's series expansion to the right-hand side of (5) but in this case, the bounds $\gamma_{1}\left(M_{n}\right), \gamma_{2}\left(M_{n}\right), \gamma_{3}\left(M_{n}\right)$ can all be replaced by constants independent of $n$ due to the fact that $\left\|\hat{\mu}-\mu_{0}\right\|_{L_{\infty}} \rightarrow 0$. Thus, we conclude that

$$
\int_{0}^{\tau}\left\{\left(\hat{\mu}(t)-\mu_{0}(t)\right)+\left(\hat{\boldsymbol{\beta}}-\boldsymbol{\beta}_{0}\right)^{T} \mathbf{Z}(t)\right\}^{2} d t \leq o_{p}\left(\frac{1}{\sqrt{n}}\right)+O_{p}\left(\frac{1}{m_{n}^{2 r}}\right) .
$$

\section{A.3. Proof of Theorem 3}

The proof of asymptotic normality is outlined as follows: We first obtain the least-favorable direction for $\boldsymbol{\beta}_{0}$ then expand the score equation for $\hat{\boldsymbol{\beta}}$ and $\hat{\mu}$ along an approximate least-favorable model. Here, the least-favorable direction for $\boldsymbol{\beta}_{0}$ is defined as a tangent function at $\mu_{0}$, denoted by $q(t)$, such that $l_{\mu}^{*} l_{\mu}[q(t)]=l_{\mu}^{*} l_{\boldsymbol{\beta}}$, where $l_{\boldsymbol{\beta}}$ is the score function for $\boldsymbol{\beta}_{0}, l_{\mu}[q(t)]$ is the score function for $\mu_{0}$ along the submodel $\mu_{0}(t)+\epsilon q(t)$, and $l_{\mu}^{*}$ is the dual operator of $l_{\mu}$. Thus, we in turn prove the following steps.

Step 1. We first show that the least-favorable direction $q(t)$ exists. Recall $H_{0}(Y)=H\left(\mu_{0}(Y)+\right.$ $\left.\boldsymbol{\beta}_{0}^{T} \mathbf{Z}(Y)\right)$ and $\Psi(Y)=\left.H^{\prime}(x)\right|_{x=\mu_{0}(Y)+\boldsymbol{\beta}_{0}^{T} \mathbf{Z}(Y)}$ By simple algebraic manipulations, we obtain that

$$
\begin{aligned}
l_{\boldsymbol{\beta}} & =\frac{\Delta \Psi(Y)}{H_{0}(Y)} \mathbf{Z}(Y)-\int_{0}^{Y} \Psi(t) \mathbf{Z}(t) d t \\
l_{\mu}[q(t)] & =\frac{\Delta \Psi(Y)}{H_{0}(Y)} q(Y)-\int_{0}^{Y} \Psi(t) q(t) d t .
\end{aligned}
$$

Moreover, the closed linear space spanned by the score functions for $\mu$ in $L_{2}(\nu)$, where $\nu$ is the dominating measure, is given by

$$
\left\{\frac{\Delta \Psi(Y)}{H_{0}(Y)} q(Y)-\int_{0}^{Y} \Psi(t) q(t) d t: q(t) \in L_{2}[0, \tau]\right\} .
$$

Thus, $l_{\mu}$ is a linear operator from $L_{2}[0, \tau]$ to $L_{2}(\nu)$. Its dual operator $l_{\mu}^{*}$ satisfies that for any $q \in L_{2}[0, \tau]$ and a measurable function $g(\Delta, Y, \mathbf{Z})$ ( $\mathbf{Z}$ abbreviates the covariate process $\{\mathbf{Z}(t): t \in[0, \tau]\})$

$$
E\left[l_{\mu}[q] g(\Delta, Y, \mathbf{Z})\right]=\int_{0}^{\tau} l_{\mu}^{*}[g(\Delta, Y, \mathbf{Z})] q(t) d t .
$$

We expand both sides, and after comparison, we obtain that

$$
\begin{aligned}
& l_{\mu}^{*}[g(\Delta, Y, \mathbf{Z})]=E_{\mathbf{Z}}\left[\frac{\Psi(t)}{H_{0}(t)} g(1, t, \mathbf{Z}) S_{C}(t \mid \mathbf{Z}) f_{T}(t \mid \mathbf{Z})\right] \\
& \quad-\left\{E_{T, \mathbf{Z}}\left[I(T \geq t) \Psi(t) g(1, T, \mathbf{Z}) S_{C}(T \mid \mathbf{Z})\right]+E_{C, \mathbf{Z}}\left[I(C \geq t) \Psi(t) g(0, C, \mathbf{Z}) S_{T}(C \mid \mathbf{Z})\right]\right\},
\end{aligned}
$$


where $S_{T}(\cdot \mid \mathbf{Z})$ and $S_{C}(\cdot \mid \mathbf{Z})$ are the conditional survival functions for $T$ and $C$ given $\mathbf{Z}$, respectively. Therefore,

$$
l_{\mu}^{*} l_{\mu}[q]=q(t) E\left[\frac{\Psi(t)^{2}}{H_{0}(t)^{2}} S_{C}(t \mid \mathbf{Z}) f_{T}(t \mid \mathbf{Z})\right]+\int q(s) k(s, t) d s,
$$

where

$$
\begin{gathered}
k(s, t)=-E_{\mathbf{Z}}\left[f_{T}(t \mid \mathbf{Z}) S_{C}(t \mid \mathbf{Z}) I(t \geq s) \Psi(s) \frac{\Psi(t)}{H_{0}(t)}\right]-E_{\mathbf{Z}}\left[f_{T}(s \mid \mathbf{Z}) S_{C}(s \mid \mathbf{Z}) I(s \geq t) \Psi(t) \frac{\Psi(s)}{H_{0}(s)}\right] \\
+E_{Y, \mathbf{Z}}[\Psi(t) \Psi(s) I(Y \geq t) I(Y \geq s)] .
\end{gathered}
$$

Note that $k(s, t)$ is a continuous function of $(t, s)$ based on (C.5). Therefore, the equation $l_{\mu}^{*} l_{\mu}[q]=l_{\mu}^{*} l_{\boldsymbol{\beta}}$ is a Fredholm-type equation and the existence of the solution is equivalent to showing that $l_{\mu}^{*} l_{\mu}[\tilde{q}]=0$ has a trivial solution. The latter is clear from the following arguments: if $l_{\mu}^{*} l_{\mu}[\tilde{q}]=0$, then $E\left\{l_{\mu}[\tilde{q}] l_{\mu}[\tilde{q}]\right\}=0$. Thus, $l_{\mu}[\tilde{q}]=0$, so it is clear that $\tilde{q}(t) \equiv 0$. We conclude that there exists a solution $q(t)$ such that $l_{\mu}^{*} l_{\mu}[q(t)]=l_{\mu}^{*} l_{\boldsymbol{\beta}}$. Clearly, from the equation for $q(t)$ and condition (C.5) as well as the smoothness condition in Theorem 2, $q(t)$ is continuously three times differentiable in $[0, \tau]$.

Step 2. We choose an approximate submodel $(\hat{\boldsymbol{\beta}}+\epsilon \mathbf{b}, \hat{\mu}+\epsilon \hat{q})$, where $\hat{q}$ is the approximate wavelet function for $q$ in the sieve space $\mathcal{S}_{n}$, and thus $\hat{q} \in W^{r, 2}$ and $\|\hat{q}-q\|_{L_{2}} \leq O\left(1 / m_{n}^{r}\right)$. Since $(\hat{\boldsymbol{\beta}}, \hat{\mu})$ maximizes the observed likelihood function along this submodel, we immediately obtain that

$$
\mathbf{P}_{n}\left\{l_{\boldsymbol{\beta}}(\hat{\boldsymbol{\beta}}, \hat{\mu})+l_{\mu}(\hat{\boldsymbol{\beta}}, \hat{\mu})[\hat{q}]\right\}=0,
$$

where $l_{\boldsymbol{\beta}}(\hat{\boldsymbol{\beta}}, \hat{\mu})$ is the score function for $\boldsymbol{\beta}$ evaluated at $(\hat{\boldsymbol{\beta}}, \hat{\mu})$ and $l_{\mu}(\hat{\boldsymbol{\beta}}, \hat{\mu})[\hat{q}]$ is the score function for $\mu$ evaluated at $(\hat{\boldsymbol{\beta}}, \hat{\mu})$. Thus,

$$
\mathbf{G}_{n}\left\{l_{\boldsymbol{\beta}}(\hat{\boldsymbol{\beta}}, \hat{\mu})+l_{\mu}(\hat{\boldsymbol{\beta}}, \hat{\mu})[\hat{q}]\right\}=-\sqrt{n} \mathbf{P}\left\{l_{\boldsymbol{\beta}}(\hat{\boldsymbol{\beta}}, \hat{\mu})+l_{\mu}(\hat{\boldsymbol{\beta}}, \hat{\mu})[\hat{q}]\right\} .
$$

Since the function $l_{\boldsymbol{\beta}}(\hat{\boldsymbol{\beta}}, \hat{\mu})+l_{\mu}(\hat{\boldsymbol{\beta}}, \hat{\mu})[\hat{q}]$ belongs to a P-Donsker class, the above equation becomes

$$
\mathbf{G}_{n}\left\{l_{\boldsymbol{\beta}}\left(\boldsymbol{\beta}_{0}, \mu_{0}\right)+l_{\mu}\left(\boldsymbol{\beta}_{0}, \mu_{0}\right)[q]\right\}+o_{p}(1)=-\sqrt{n} \mathbf{P}\left\{l_{\boldsymbol{\beta}}(\hat{\boldsymbol{\beta}}, \hat{\mu})+l_{\mu}(\hat{\boldsymbol{\beta}}, \hat{\mu})[\hat{q}]\right\} .
$$

We perform a Taylor's series expansion of the right-hand side at $\left(\boldsymbol{\beta}_{0}, \mu_{0}\right)$, and obtain

$$
\begin{gathered}
\mathbf{G}_{n}\left\{l_{\boldsymbol{\beta}}\left(\boldsymbol{\beta}_{0}, \mu_{0}\right)+l_{\mu}\left(\boldsymbol{\beta}_{0}, \mu_{0}\right)[q]\right\}+o_{p}(1)=-\sqrt{n} \mathbf{P}\left\{l_{\boldsymbol{\beta} \boldsymbol{\beta}}\left(\boldsymbol{\beta}_{0}, \mu_{0}\right)+l_{\boldsymbol{\beta}_{\mu}}\left(\boldsymbol{\beta}_{0}, \mu_{0}\right)[q]\right\}\left(\hat{\boldsymbol{\beta}}-\boldsymbol{\beta}_{0}\right) \\
-\sqrt{n} \mathbf{P}\left\{l_{\boldsymbol{\beta}_{\mu}}\left(\boldsymbol{\beta}_{0}, \mu_{0}\right)\left[\hat{\mu}-\mu_{0}\right]+l_{\mu \mu}\left(\boldsymbol{\beta}_{0}, \mu_{0}\right)\left[q, \hat{\mu}-\mu_{0}\right]\right\}+\sqrt{n} O\left(\left|\hat{\boldsymbol{\beta}}-\boldsymbol{\beta}_{0}\right|^{2}+\left\|\hat{\mu}-\mu_{0}\right\|_{L_{2}}^{2}+\|\hat{q}-q\|_{L_{2}}^{2}\right) .
\end{gathered}
$$


Here, $l_{\boldsymbol{\beta}_{\mu}}\left(\boldsymbol{\beta}_{0}, \mu_{0}\right)\left[\hat{\mu}-\mu_{0}\right]$ is the derivative of $l_{\boldsymbol{\beta}}$ along the path $\boldsymbol{\beta}=\boldsymbol{\beta}_{0}, \mu=\mu_{0}+\epsilon\left(\hat{\mu}-\mu_{0}\right)$ and $l_{\mu \mu}\left(\boldsymbol{\beta}_{0}, \mu_{0}\right)\left[q, \hat{\mu}-\mu_{0}\right]$ is the derivative of $l_{\mu}[q]$ along the path $\boldsymbol{\beta}=\boldsymbol{\beta}_{0}, \mu=\mu_{0}+\epsilon\left(\hat{\mu}-\mu_{0}\right)$. The second term on the right-hand side of (7) is zero since $q(t)$ satisfies $l_{\mu}^{*} l_{\mu}[q(t)]=l_{\mu}^{*} l_{\boldsymbol{\beta}}$; the third term on the right-hand side of $(7)$ is $o_{p}(1)$ based on the results of the convergence rate for $(\hat{\boldsymbol{\beta}}, \hat{\mu})$, and the condition that $\sqrt{n} / m_{n}^{2 r} \rightarrow 0$. Hence,

$$
-\sqrt{n} \mathbf{P}\left\{l_{\boldsymbol{\beta} \boldsymbol{\beta}}\left(\boldsymbol{\beta}_{0}, \mu_{0}\right)+l_{\boldsymbol{\beta}_{\mu}}\left(\boldsymbol{\beta}_{0}, \mu_{0}\right)[q]\right\}\left(\hat{\boldsymbol{\beta}}-\boldsymbol{\beta}_{0}\right)=\mathbf{G}_{n}\left\{l_{\boldsymbol{\beta}}\left(\boldsymbol{\beta}_{0}, \mu_{0}\right)+l_{\mu}\left(\boldsymbol{\beta}_{0}, \mu_{0}\right)[q]\right\}+o_{p}(1) .
$$

Step 3. We show that the matrix $\mathbf{P}\left\{l_{\boldsymbol{\beta} \boldsymbol{\beta}}\left(\boldsymbol{\beta}_{0}, \mu_{0}\right)+l_{\boldsymbol{\beta}_{\mu}}\left(\boldsymbol{\beta}_{0}, \mu_{0}\right)[q]\right\}$ is non-singular. If not, there exists a non-zero vector $\mathbf{b}$ such that

$$
\mathbf{b}^{T} \mathbf{P}\left\{l_{\boldsymbol{\beta} \boldsymbol{\beta}}\left(\boldsymbol{\beta}_{0}, \mu_{0}\right)+l_{\boldsymbol{\beta}_{\mu}}\left(\boldsymbol{\beta}_{0}, \mu_{0}\right)[q]\right\} b=0 .
$$

That is, $\mathbf{P}\left[\left\{\mathbf{b}^{T} l_{\boldsymbol{\beta}}+\mathbf{b}^{T} l_{\mu}[q]\right\}^{2}\right]=0$. Then, $\mathbf{b}^{T} l_{\boldsymbol{\beta}}+\mathbf{b}^{T} l_{\mu}[q]=0$. It is easy to see that $\mathbf{b}^{T} \mathbf{Z}(t)+$ $q(t)=0$. We thus obtain a contradiction.

Step 4. Finally, from (8), we obtain that

$$
\sqrt{n}\left(\hat{\boldsymbol{\beta}}-\boldsymbol{\beta}_{0}\right)=-\left[\mathbf{P}\left\{l_{\boldsymbol{\beta} \boldsymbol{\beta}}\left(\boldsymbol{\beta}_{0}, \mu_{0}\right)+l_{\boldsymbol{\beta}_{\mu}}\left(\boldsymbol{\beta}_{0}, \mu_{0}\right)[q]\right\}\right]^{-1} \mathbf{G}_{n}\left\{l_{\boldsymbol{\beta}}\left(\boldsymbol{\beta}_{0}, \mu_{0}\right)+l_{\mu}\left(\boldsymbol{\beta}_{0}, \mu_{0}\right)[q]\right\}+o_{p}(1) .
$$

Therefore, $\sqrt{n}\left(\hat{\boldsymbol{\beta}}-\boldsymbol{\beta}_{0}\right)$ converges to a normal distribution and has influence function given by

$$
\left[\mathbf{P}\left\{l_{\boldsymbol{\beta} \boldsymbol{\beta}}\left(\boldsymbol{\beta}_{0}, \mu_{0}\right)+l_{\boldsymbol{\beta}_{\mu}}\left(\boldsymbol{\beta}_{0}, \mu_{0}\right)[q]\right\}\right]^{-1}\left\{l_{\boldsymbol{\beta}}\left(\boldsymbol{\beta}_{0}, \mu_{0}\right)+l_{\mu}\left(\boldsymbol{\beta}_{0}, \mu_{0}\right)[q]\right\} .
$$

Since this influence function is on the linear space spanned by the score functions $l_{\boldsymbol{\beta}}$ and $l_{\mu}[q]$, the influence function is the same as the efficient influence function for $\boldsymbol{\beta}_{0}$. Hence, the asymptotic variance of $\sqrt{n}\left(\hat{\boldsymbol{\beta}}-\boldsymbol{\beta}_{0}\right)$ attains the semiparametric efficiency bound. 


\section{REFERENCES}

Adams, R. A. (1975), Sobolev Spaces, New York: Academic Press.

Andersen, P. K. and Gill, R. D. (1982), "Cox's regression model for counting processes: a large-sample study," Annals of Statistics, 10, 1100-1120.

Bickle, P.J., Klaassen, C.A.I., Ritov, Y. and Wellner, J.A. (1993), Efficient and Adaptive Estimation for Semiparametric Models, Baltimore: John-Hopkins University Press.

Box, G. E. P. and Cox, D. R. (1964), "An Analysis of Transformations (with discussion)," Journal of the Royal Statistical Society Series B, 26, 211-252.

Coleman, T.F. and Li, Y. (1994), "On the convergence reflective Newton methods for large-scale nonlinear minimization subject to bounds," Mathematical Programming, 67, 189-224.

Coleman, T.F. and Li, Y. (1996), "An interior, trust region approach for nonlinear minimization subject to bounds," SIAM Journal on Optimization, 6, 418-445.

Cox, D. R. (1972), "Regression models and life-tables (with discussion)," Journal of the Royal Statistical Society Series B, 34, 187-220.

Cox, D. R. (1975), "Partial likelihood," Biometrika, 62, 269-276.

Daubechies, I. (1992), Ten Lectures on Wavelets, Notes from the 1990 CBMS-NSF Conference on Wavelets and Applications at Lowell, MA. SIAM, Philadelphia, PA.

Fan, J. and Wong, W. H. (2000), "Comment on On profile likelihood by Murphy and van der Vaart," Journal of the American Statistical Association, 92, 968-976.

Fan, J. and Zhang, J. (2004), "Sieve empirical likelihood ratio tests for nonparametric functions," Annals of Statistics, to appear.

Härdle, W., Kerkyacharian, G., Picard, D., and Tsybakov, A. (2000), Wavelets, Approximation, and Statistical Applications, Lecture Notes in Statistics 129, New York: Springer.

Lin, D. Y. and Ying, Z. (1994), "Semiparametric analysis of the additive risk model," Biometrika, $81,61-71$. 
Lin, D.Y. and Ying, Z. (1995), "Semiparametric analysis of general additive-multiplicative hazard models for counting processes," Annals of Statistics, 23, 1712-1734.

Mallat, S. (1998), A Wavelet Tour of Signal Processing, New York: Academic Press.

Murphy, S. A. and van der Vaart, A. W. (2000), "On profile likelihood," Journal of the American Statistical Association, 95, 449-465.

Shen, X. (1997), "On methods of sieves and penalization," Annals of Statistics, 25, 2555-2591.

Shen, X. (1998), "Proportional odds regression and sieve maximum likelihood estimation," Biometrika, 85, 165-177.

Shen, X. and Shi, J. (2004), "Sieve likelihood ratio inference on general parameter space," Science China, to appear.

Shen, X. and Wong, W. H. (1994), "Convergence rate of sieve estimates," Annals of Statistics, $22,580-615$.

Socinski, M. A., Schell, M. J., Peterman, A., Bakri, K., Yates, S., Gitten, R., Unger, P., Lee, J., Lee, J. H., Tynan, M., Moore, M. and Kies, M. S. (2002), "Phase III trial comparing a defined duration of therapy versus continuous therapy followed by second-line therapy in advanced-stage IIIB/IV non-small-cell lung cancer," Journal of Clinical Oncology, 20, $1335-1343$.

van der Vaart, A. W. (1998), Asymptotic Statistics, New York: Cambridge University Press.

van der Vaart, A. W. and Wellner, J. A. (1995), Weak Convergence and Empirical Processes, New York: Springer. 
Table 1: Simulation Results from 500 Repetitions

\begin{tabular}{cccccccc}
\hline$s$ & $n$ & Coef. & True value & Estimate & SE & Est. SE & CP \\
\hline \hline 0 & 200 & $\beta_{1}$ & 0.7 & 0.703 & 0.236 & 0.234 & 0.948 \\
& & $\beta_{2}$ & 0.2 & 0.207 & 0.156 & 0.151 & 0.942 \\
& 400 & $\beta_{1}$ & 0.7 & 0.701 & 0.160 & 0.165 & 0.966 \\
& & $\beta_{2}$ & 0.2 & 0.192 & 0.108 & 0.107 & 0.936 \\
& & & & & & & \\
0.25 & 200 & $\beta_{1}$ & 0.7 & 0.691 & 0.260 & 0.278 & 0.968 \\
& & $\beta_{2}$ & 0.2 & 0.203 & 0.182 & 0.179 & 0.960 \\
& 400 & $\beta_{1}$ & 0.7 & 0.708 & 0.190 & 0.194 & 0.956 \\
& & $\beta_{2}$ & 0.2 & 0.195 & 0.126 & 0.125 & 0.956 \\
& & & & & & & \\
0.5 & \multirow{2}{*}{200} & $\beta_{1}$ & 0.7 & 0.708 & 0.327 & 0.317 & 0.930 \\
& & $\beta_{2}$ & 0.2 & 0.210 & 0.207 & 0.203 & 0.948 \\
& 400 & $\beta_{1}$ & 0.7 & 0.691 & 0.224 & 0.222 & 0.936 \\
& & $\beta_{2}$ & 0.2 & 0.194 & 0.136 & 0.142 & 0.956 \\
& & & & & & & \\
0.75 & 200 & $\beta_{1}$ & 0.7 & 0.678 & 0.356 & 0.349 & 0.936 \\
& & $\beta_{2}$ & 0.2 & 0.191 & 0.212 & 0.222 & 0.964 \\
& 400 & $\beta_{1}$ & 0.7 & 0.693 & 0.251 & 0.249 & 0.950 \\
& & $\beta_{2}$ & 0.2 & 0.208 & 0.166 & 0.158 & 0.950 \\
& & & & & & & \\
1 & 200 & $\beta_{1}$ & 0.7 & 0.735 & 0.384 & 0.379 & 0.944 \\
& & $\beta_{2}$ & 0.2 & 0.172 & 0.254 & 0.241 & 0.928 \\
& 400 & $\beta_{1}$ & 0.7 & 0.695 & 0.286 & 0.273 & 0.934 \\
& & $\beta_{2}$ & 0.2 & 0.203 & 0.170 & 0.173 & 0.960 \\
\hline
\end{tabular}

NOTE. SE is the standard error, Est. SE is the average of the profile likelihood estimated standard errors, and CP is the $95 \%$ coverage proportion, respectively. 
(a)

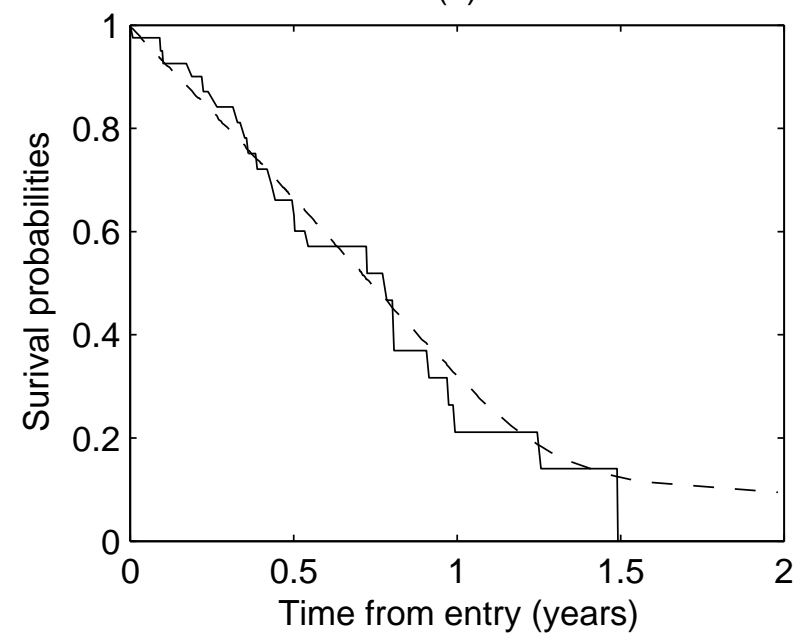

(c)

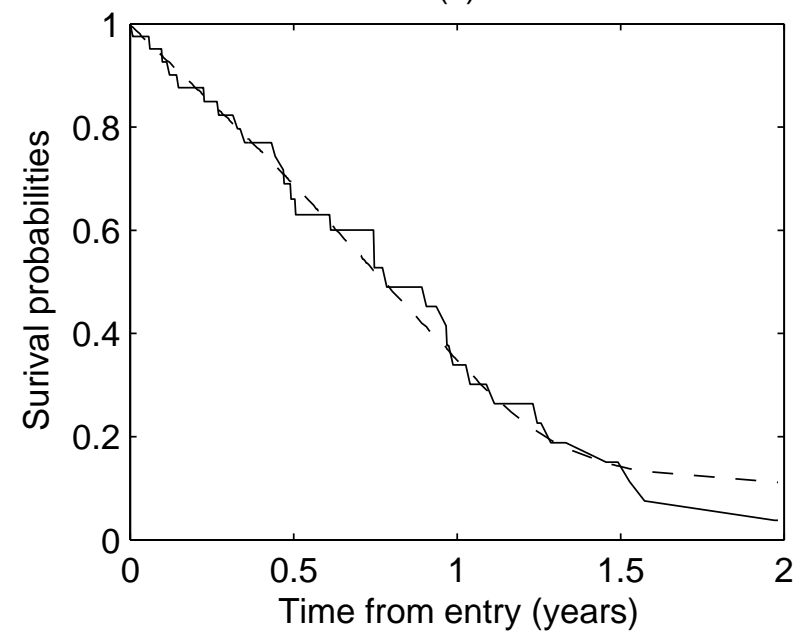

(b)

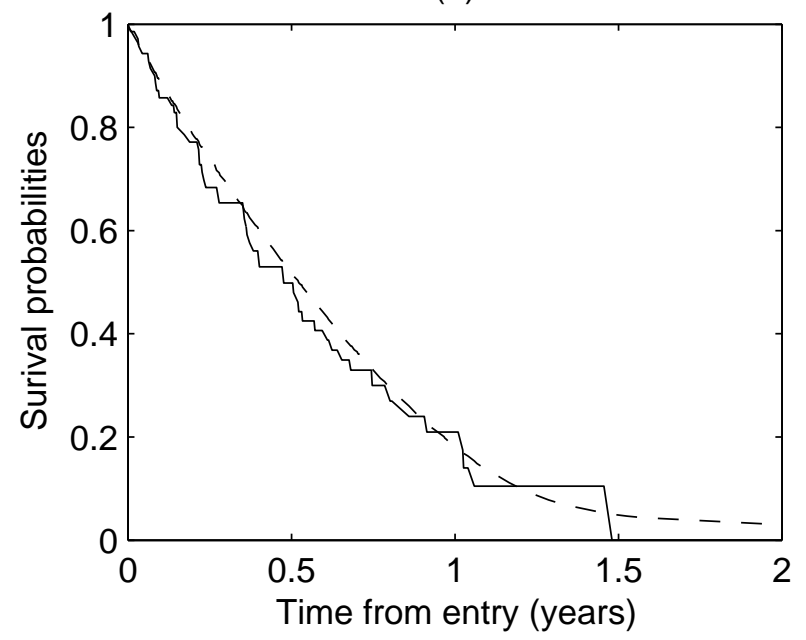

(d)

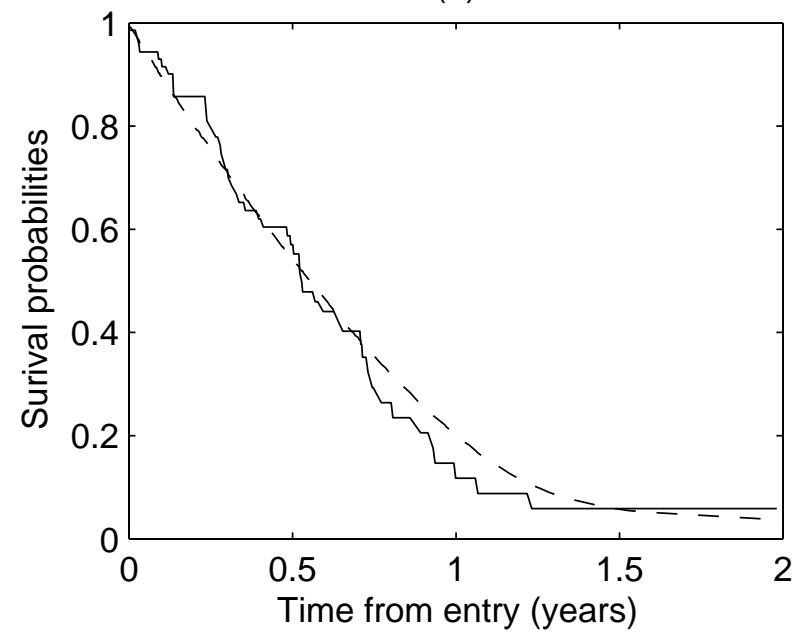

Figure 1: Predicated survival curves (dashed lines) based on the best model vs the KaplanMeier curves (solid lines): (a) Arm A, female; (b) Arm A, male; (c) Arm B, female; (d) Arm $\mathrm{B}$, male. 